\title{
ANDROID APPLICATION TO LOCATE AND TRACK MOBILE PHONES(AALTM)- AN IMPLEMENTATION OF LOCATION-BASED SERVICES
}

\author{
Lovely June Sanglyne ${ }^{1}$ \\ ${ }^{1}$ MCA Student, Department of Computer Science \& Engineering and Information Technology, School of Technology- \\ Assam Don Bosco University, Assam, India
}

\begin{abstract}
With the advancement of technology and modern science, people are expecting information about the location of any object for tracking purposes. GPS is a system which has been implemented and is accessible without any restriction. Having the facility of GPS, we need a GPS device to calculate the location from the information taken from GPS. This paper presents a study on Location Based Services and is directed on locating and tracking of Android devices. In this implementation I am using Global Positioning System (GPS) and Web Services, and android smart phone device since Android devices have a built-in feature of GPS service. Some latest demanding tools and technology used for this implementation are Java, AVD, WAMP etc.
\end{abstract}

Keywords: Location Based Services; Global Positioning System (GPS); location; path

\section{INTRODUCTION}

A Location-Based Service (LBS) provides information and service of the geographical position of the mobile device .This can be done through the mobile network and utilizing the ability to make use of that information .In order to determine the location of user's mobile device in a LBS application , positioning technologies or a positioning component such as satellite positioning, cellular network positioning, WLAN stations or radio beacons is required. Hence most of the current LBS services do not require users to input location manually, such as providing street name or zip code.

Service providers are also required since they offer different kinds of LBS services to users and are responsible for processing service requests and responds back by sending request results. Servers can calculate locations or position, search for a path or route travelled, or search specific information regarding user's position. Usually all the information requested by users are not store and maintain by the Service providers, geographic data are collect and stored by content provider. Informations such as Locationbased and other related data are also collect and store by the content provider. Service servers will requests and process these data and then returned to users.

Some examples of location based services [2] are:

- $\quad$ To determine the nearest business or service, such as an Bank ,Restaurants, Hotels, etc

- Receiving alerts or notifications, such as notification of Sale in Shopping Malls or news of Traffic Jam in nearby area.

- Searching friends or tracking the location of the stolen phone or lost phone.

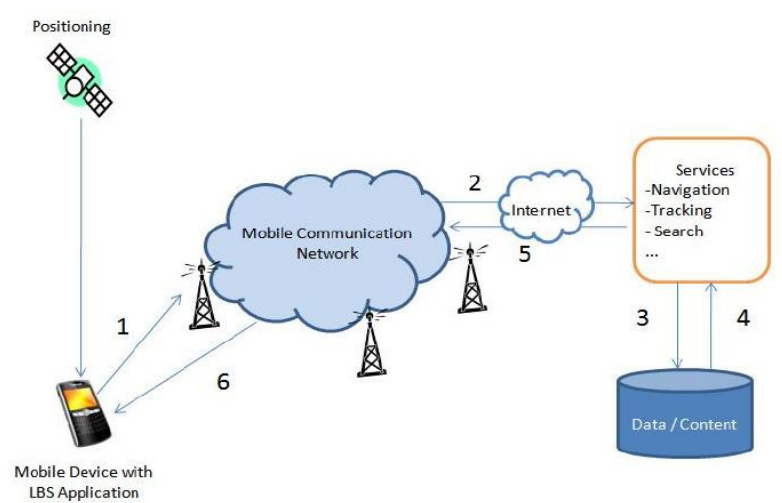

Fig 1 LBS components and Service Process

\section{ARCHITECTURE OF LOCATION BASED SERVICES}

The architecture of LBS can be generalized as shown below:

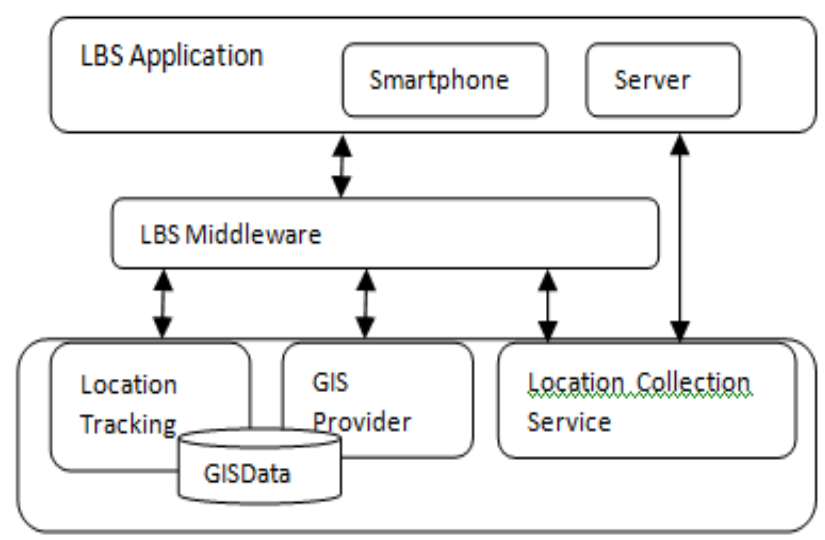

Fig 2 Architecture of the Location Based Services 
The architecture of LBS consists of five components:

1) LBS Application: This represents some application such This consists of Smartphone component, which has a number of sensors, and potentially a server component that includes application-specific data (such as location-tagged information).

2) LBS Middleware: The Core LBS Features such as Location Tracking, GIS Provider and Location Collection Services are accessible through a consistent interface provided by the LBS Middleware to the LBS applications.

3) Location Tracking: This component stores the location trace of individual users and contains the data that allows a user's route to be determined and potentially predicted.

4) GIS (Geographic Information System) Provider: This component provides geospatial functionality for many LBSs such as map information, map visualization and directory services. Google Maps with its API can be considered a GIS provider.

5) For many LBSs ,the geospatial functionality such as map information, map visualization and directory services are being provided by the GIS provider. Google Maps with its API can be considered a GIS provider.

6) Location Collection Service: To get a latitude and longitude for a specific user,the Location Collection Service is use. The Location Collection Service component is accessible via the LBS Middleware (such as mobile network triangulation via a service provider) or directly (e.g., via GPS receiver in the Smartphone) depending on the technology.

\section{ELEMENTS OF LOCATION-BASED SERVICES MENTS OF LOCATION-BASED SERVICES}

Location-based services is used to describe the different technologies used to find a device current location. Android provides access to the above components to facilitate the implementation of LBS services through the help of the four main LBS elements.

The different technologies used to find a device current location can be described by using a Location-based service(s). The four main LBS elements facilitates the implementation of LBS services components (mentioned in section A) which is accessible through Android.

This section explains the four main LBS elements:

1) Location Manager: This element provides access to the location-based services. The following are some of the application of the location Manager :

- Obtain the current Location

- Track movement .

- Detect movement into and out of a specified area.

- $\quad$ Find available Location Providers.
2) Location Providers: These represent different technology which are used to determine the device's current location or location-finding and provides periodic reports on the geographical location. It also determines the physical location, that is, handle GIS. , Location Provider component of Android application facilitates the determination of the available provider(s) and the selection of appropriate one among these available provider(s).

The static variables defined in the Location Manager class can be accessed directly by GPS provider and Network provider.GPS and network providers are two different ways to get Android device location (latitude and longitude). Both have their own advantages and we may have to use both synchronous.

- $\quad$ GPS gives the exact location of where we are standing .However in indoor situations GPS may not provide the location quickly .

- Network location provider use mobile connectivity provider and will give the nearest tower location.

- If the GPS is not enabled passive provider return coarse fixes.

3) Geocoding: Geocoding can be done in two different ways. That is, reverse geocoding can forward geocoding. Conversion of geographical coordinates (longitude, latitude) into street address can be done using reverse geocoding whereas conversion of street address into geographical coordinates can be done using reverse geocoding. Reverse geocoding uses getLatitude() and getLongitude () to get the geographical coordinates.

4) Google-Map: Android provides a number of objects to handle maps in LBS system like MapView which displays the map.

\section{OBJECTIVES}

The objective of the Real Time Location Tracking system is to serve people a more effective location based tracking facility.

In real life, this system is helpful in situations such as the managing a group of people going for a trip or a tour whereby the management is little tough because it is quite impossible to restrict the movement of all participants. This system can help the management team to track their previous location and where they are now.

\section{FUNCTIONS OF THE SYSYTEM}

The Location-based tracking system using GPS and Web services via Android devices consists of two sections:

\subsection{Smartphone User Function}

The client has the ability to acquired his/her location and record his/her footprints on a website that is monitored by the administrator on the server side. 
The following are the facilities for each user.

- Registration.

- Acquire his/her location.

His/her location will be automatically record on a website.

\subsection{Administrator Function}

The administrator function has the facility on his PC to "sit and observe" the location of multiple users. The administrator has the ability to track the location the user had visited, and view the path displayed on the Google Map. That is, monitoring the recorded footprints of each user individually on a computer screen. The application provides the administrator with the most recent location update. Unnecessary records can also be removed by the administrator.

The following are the facilities for an admin

- Login/Logout.

- Delete or remove user(s) records whenever necessary.

- Can access the user(s) location.

- Can access the user travelling path.

\section{REQUIREMENT ANALYSIS}

Table 1: Software Requirements

\begin{tabular}{|l|l|}
\hline Developer End & Deployment End \\
\hline $\begin{array}{l}\text { Java Development } \\
\text { Kit(JDK) }\end{array}$ & \\
\hline IDE: Eclipse & $\begin{array}{l}\text { Android OS: Gingerbread \& } \\
\text { Above }\end{array}$ \\
\hline Android SDK 4.2.2 & Virtual Android(Emulator) \\
\hline MYSQL Database & Google Play Service API \\
\hline
\end{tabular}

Table 2: Hardware Requirements

\begin{tabular}{|l|l|}
\hline System & Tablet \\
\hline Processor: P IV or higher. & $\begin{array}{l}\text { Android based tablet/ } \\
\text { Smartphone }\end{array}$ \\
\hline RAM 512 MB free space. & \\
\hline $\begin{array}{l}\text { Hard disk: 2.0 GB free hard } \\
\text { drive space. }\end{array}$ & \\
\hline Standard Keyboard & \\
\hline Monitor & \\
\hline
\end{tabular}

\section{SYSYTEM DESIGN}

\subsection{Modular Design}

The whole system is divided into two parts, the user and the admin section. The following are the modular designs for these parts of the system .

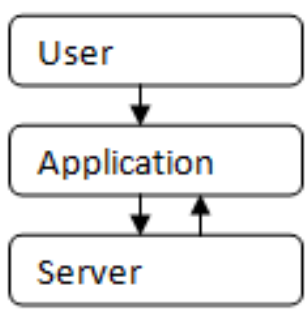

Fig. 3.(a) Modular Design for user

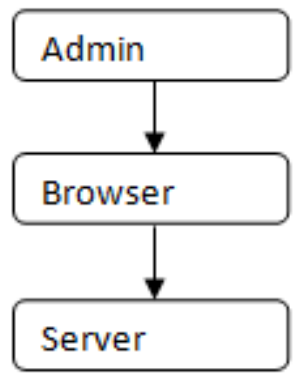

Fig.3.(b) Modular Design for Admin

\subsection{Use Case Diagram}

There are two components that helps understand the use case diagram. Those are,

- Actor: represents a user using the system. An actor in the use case diagram specifies a role played by a user or any other system that interacts with the subject.

- Use-case: represents the set of acts that a user might perform while using the system. They are used to describe characteristic or properties of an entity.
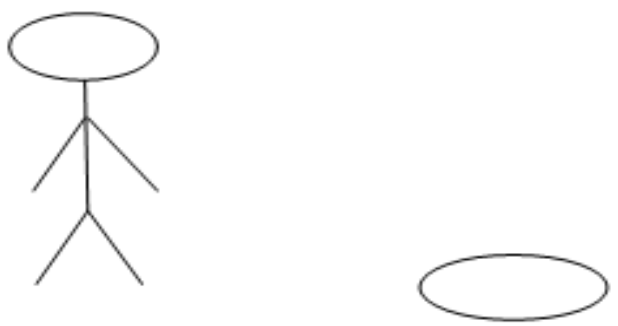

Fig.4.Symbol of Actor and use-case

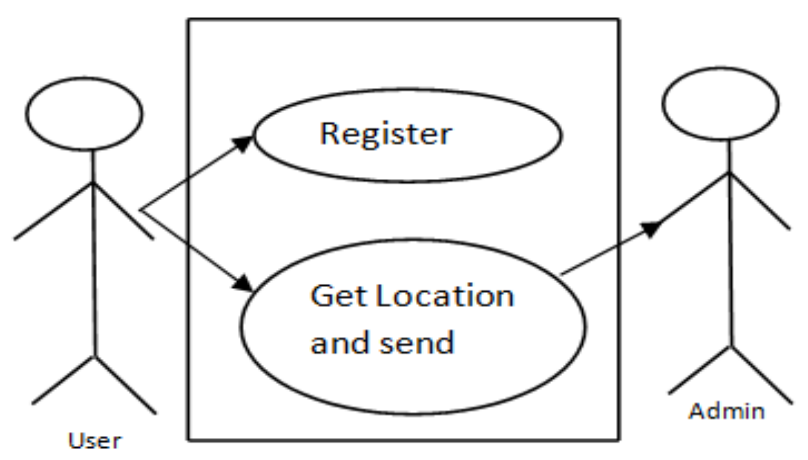

Fig.5(a). Use case Diagram for a user. 


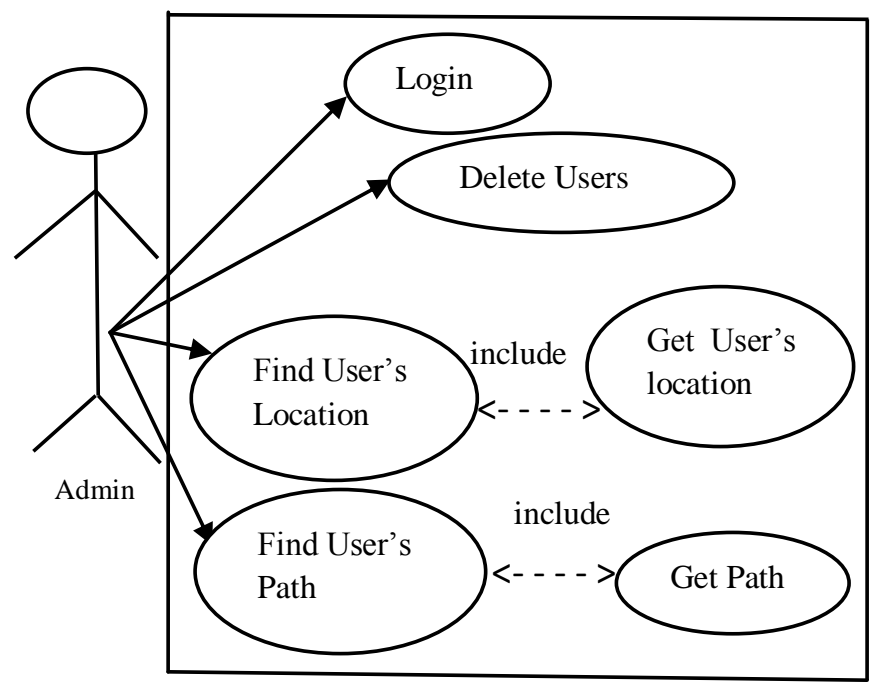

Fig.5(b). Use case Diagram for a Admin.

\subsection{Data Flow Diagram}

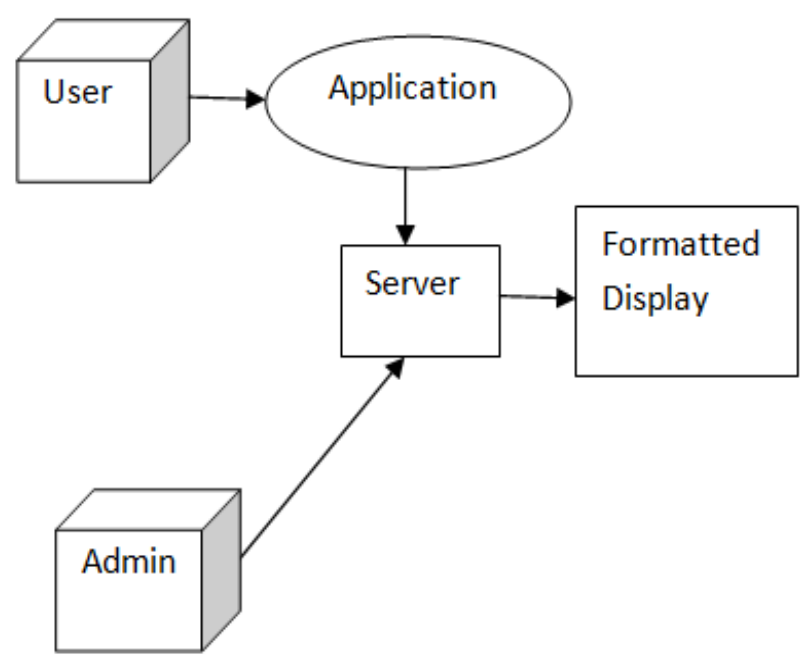

Fig.6(a). Level-0 Data Flow Diagram.

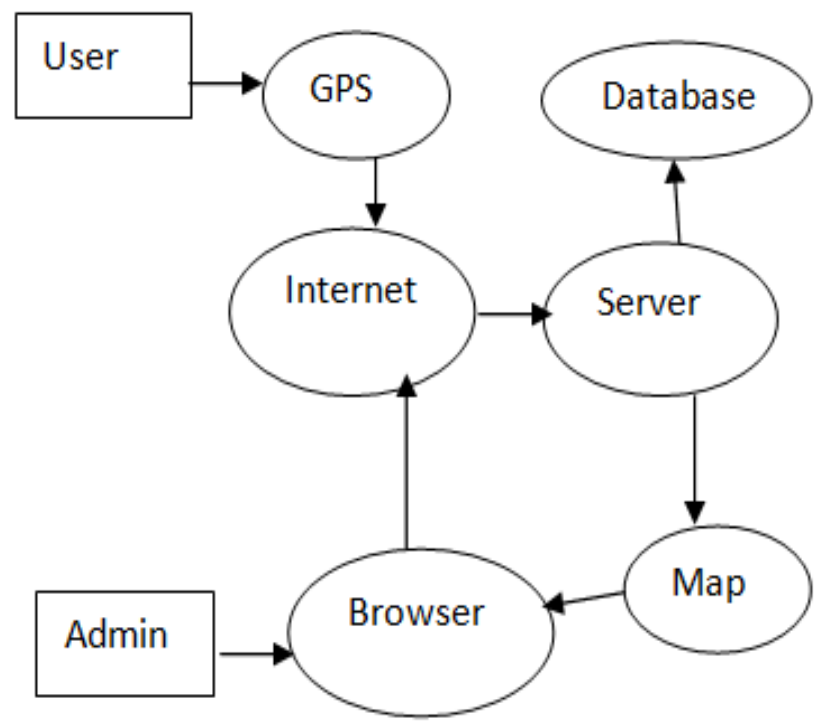

Fig.6(b). Level-1 Data Flow Diagram.

\subsection{State Diagram Design}

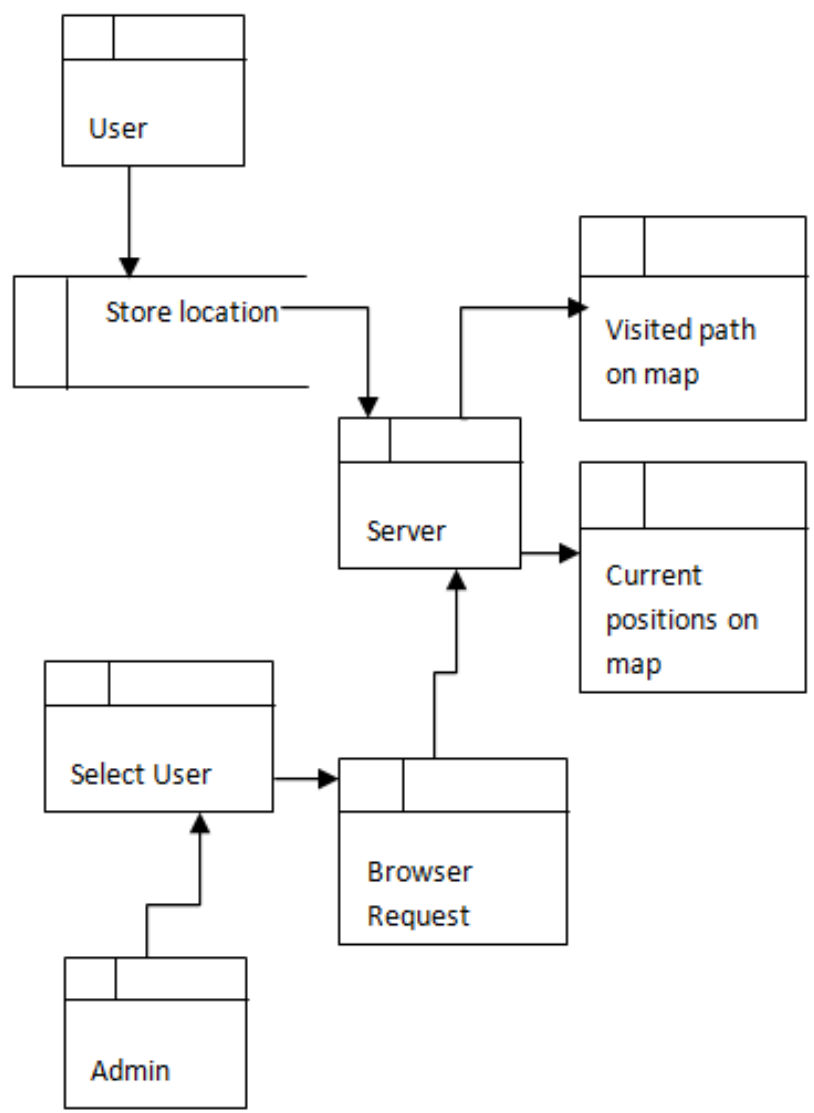

Fig.7. State Diagram

\section{IMPLEMENTATION AND RESULTS}

The application has been tested in different android devices. The data is accessing and sending to the web server upon the availability of Internet connection. This application works even if the GPS setting is off, but internet connection should be available. These are happening on background without any notification to the user.

\subsection{Snapshots for the Client (Smartphone User Function)}

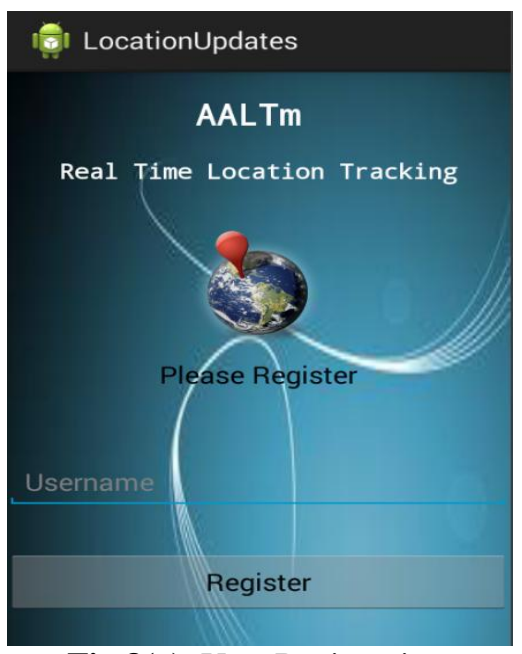

Fig.8(a). User Registration. 
The client can register by providing a Username when the application is run for the first time. The next time the user wants to run the application, this interface(Figure 8(a)) will not be visible anymore since it is running in the background.

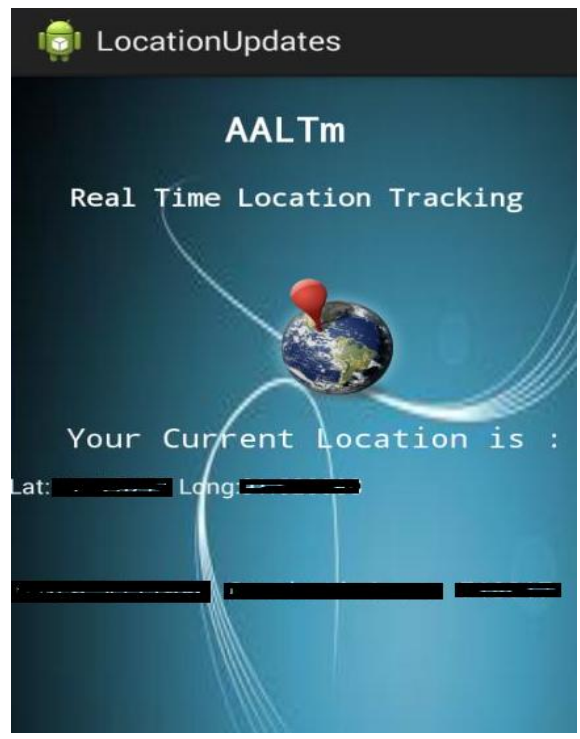

Fig.8(b). Getting location by the user.

Figure 8(b) displays the current location of the user. It includes the geographical locations (Latitude and Longitude) and street address.

\subsection{Snapshots for the Server (Administrative}

\section{Function)}

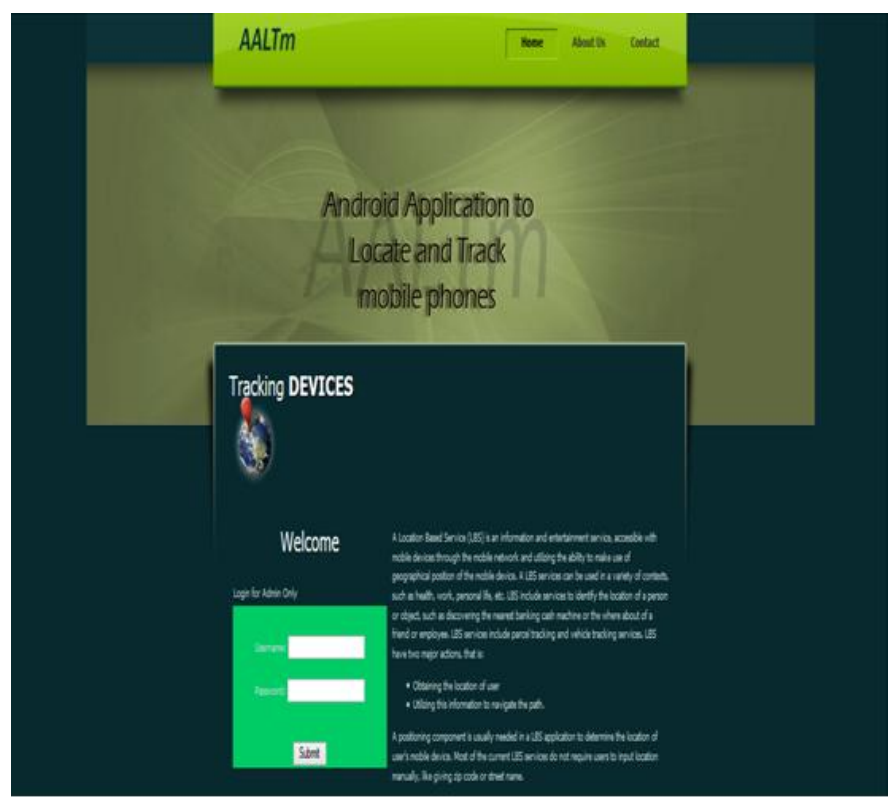

Fig.9 (a). Login Page for the Administrator.

In Figure 9(a) we can see the login page or the home page for the admin which is at the server side. The admin has the authority to login and view or delete user(s) location's details when required. The user(s) location stored in the database will be displayed on the Google map.

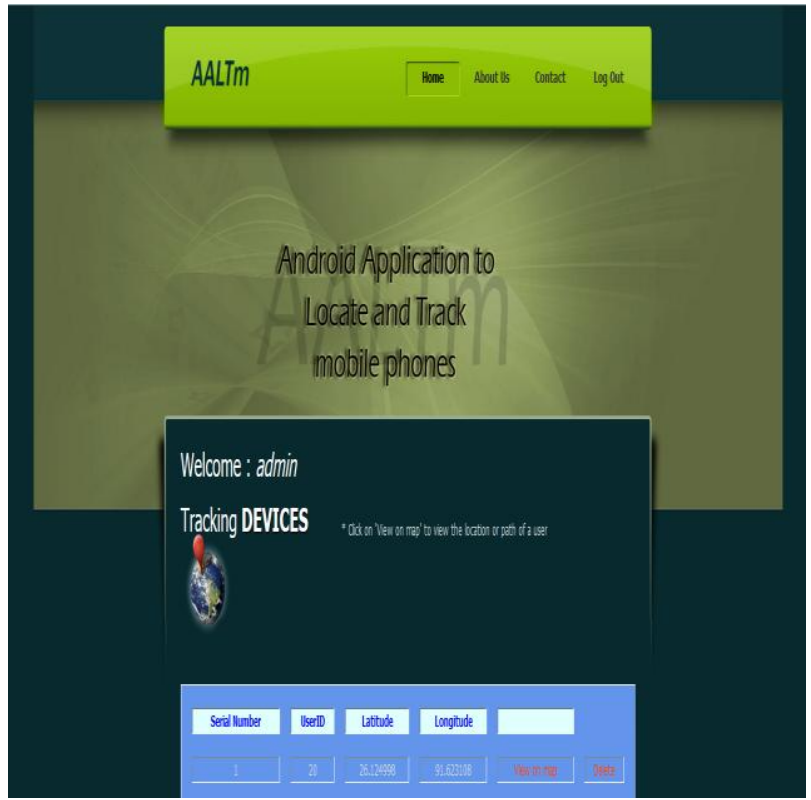

Fig.9(b).Record of a user.

Figure 9(b) and 9(c) displays all records available in the database. The information includes the serial number and the current location of the user such as Latitude, Longitude. Each record has a unique Id number. In Figure 9(b) only one record of a user is being displayed since there is only user being monitor by the admin. If there is more than one record then all the records with unique ID will be displayed as shown in Figure 9(c).

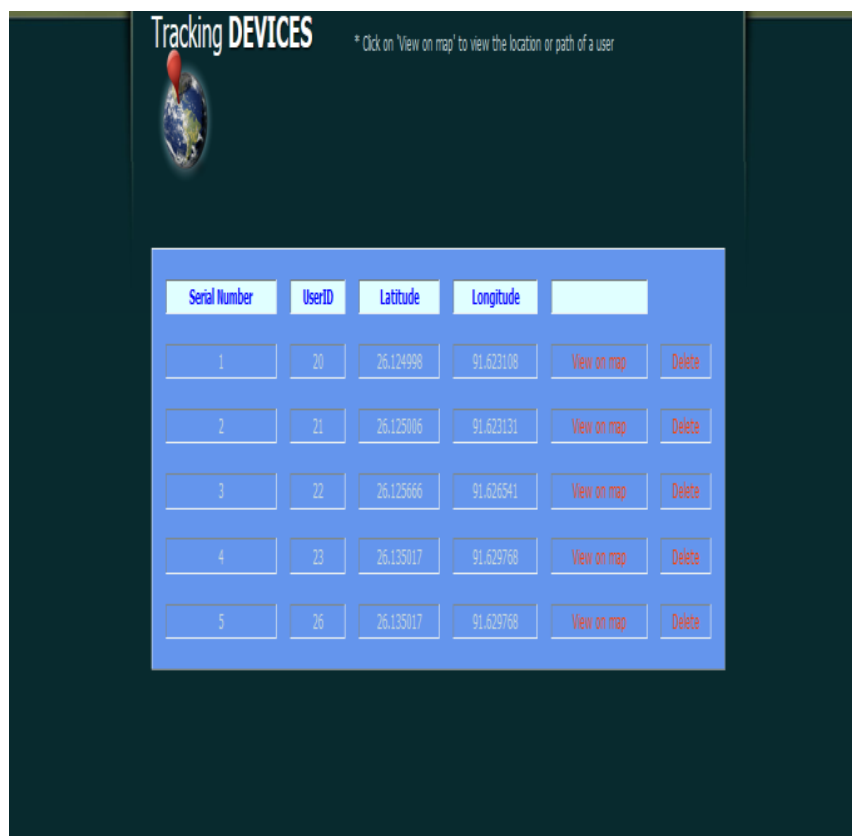

Fig.9(c).Records of multiple users .

One of the administrative function is to delete record(s).For example, as shown in figure 9(d), there are five records of users with unique UserID. The admin can delete the record of a particular user by clicking on the 'Delete' as shown in the rightmost label. Figure 9(e) shows the remaining records after deleting one record. 


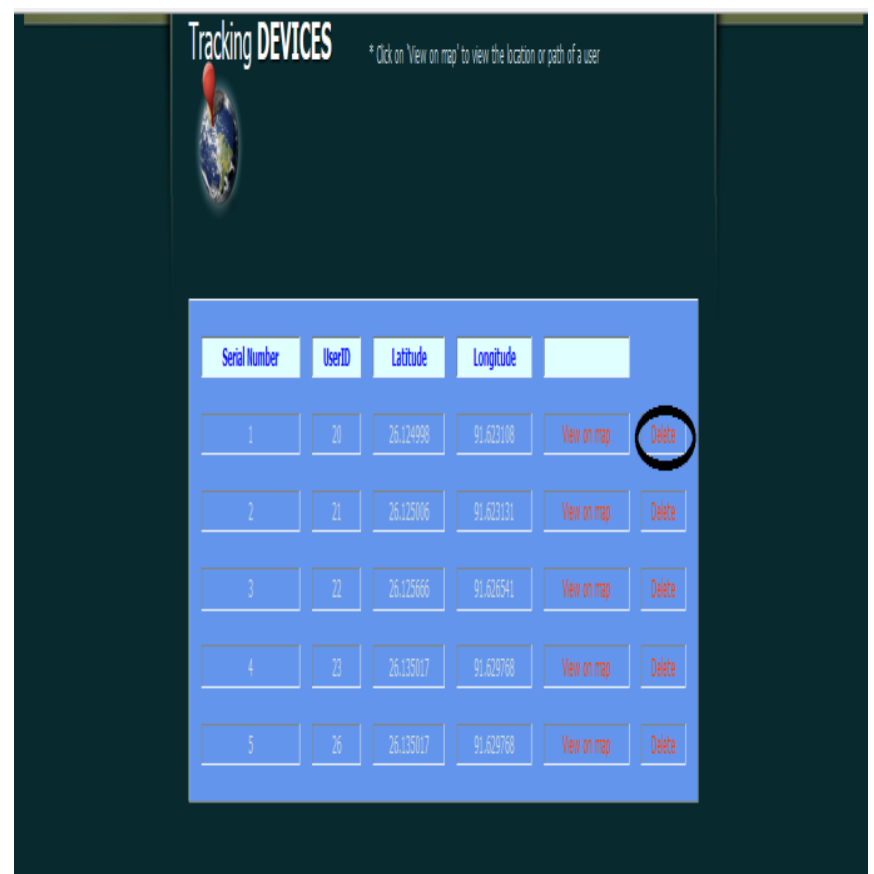

Fig.9(d).Deleting record.

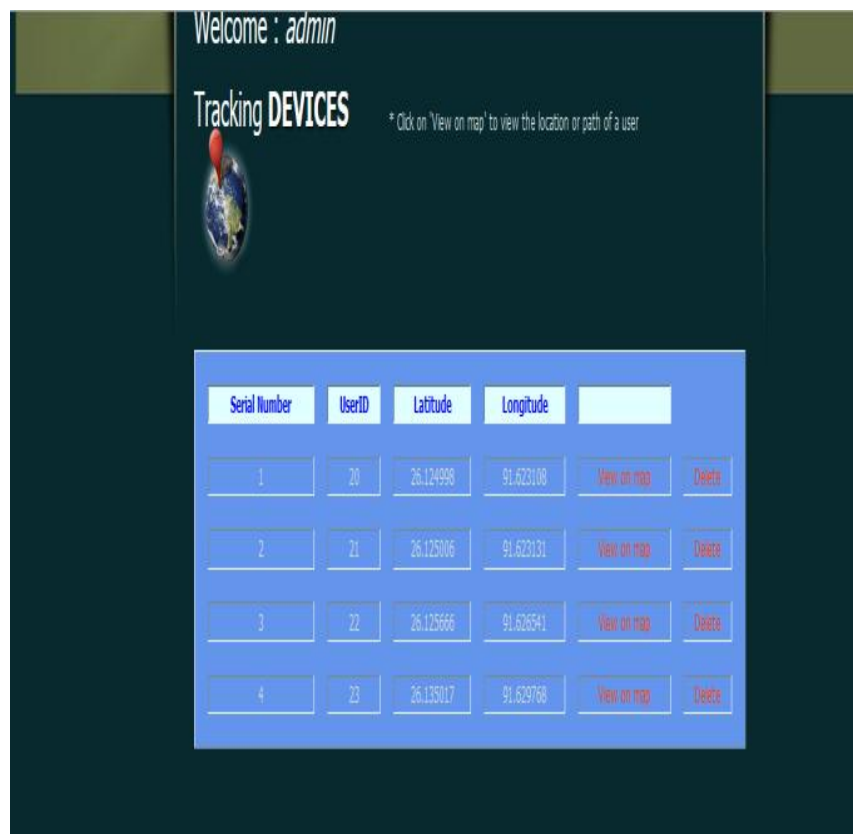

Fig.9(e.)After deleting one record.

Another administrative function is to access the location of each user and their travelled path. The admin can click on the provided link (see Figure 9(f))to view the user location(s) on the map.As shown in figure $9(\mathrm{~g})$, the location visited by the user is shown in the map by a marker point, for each location. The travelled path is shown by a line joining the markers in the map. The exact location visited by the user according to the date and time is shown in the table below the map. This location will change and update accordingly when the user changed the location or moved from one place to another.

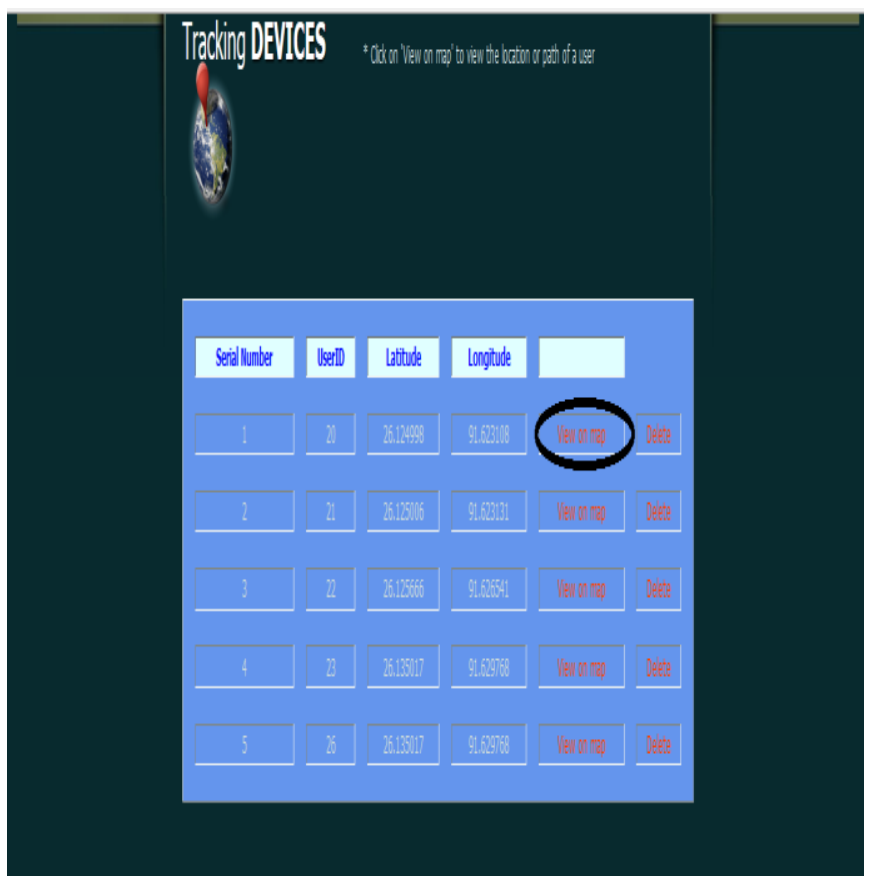

Fig.9(f). Clicking the 'view on map' to access Location of a user in a Google Map.

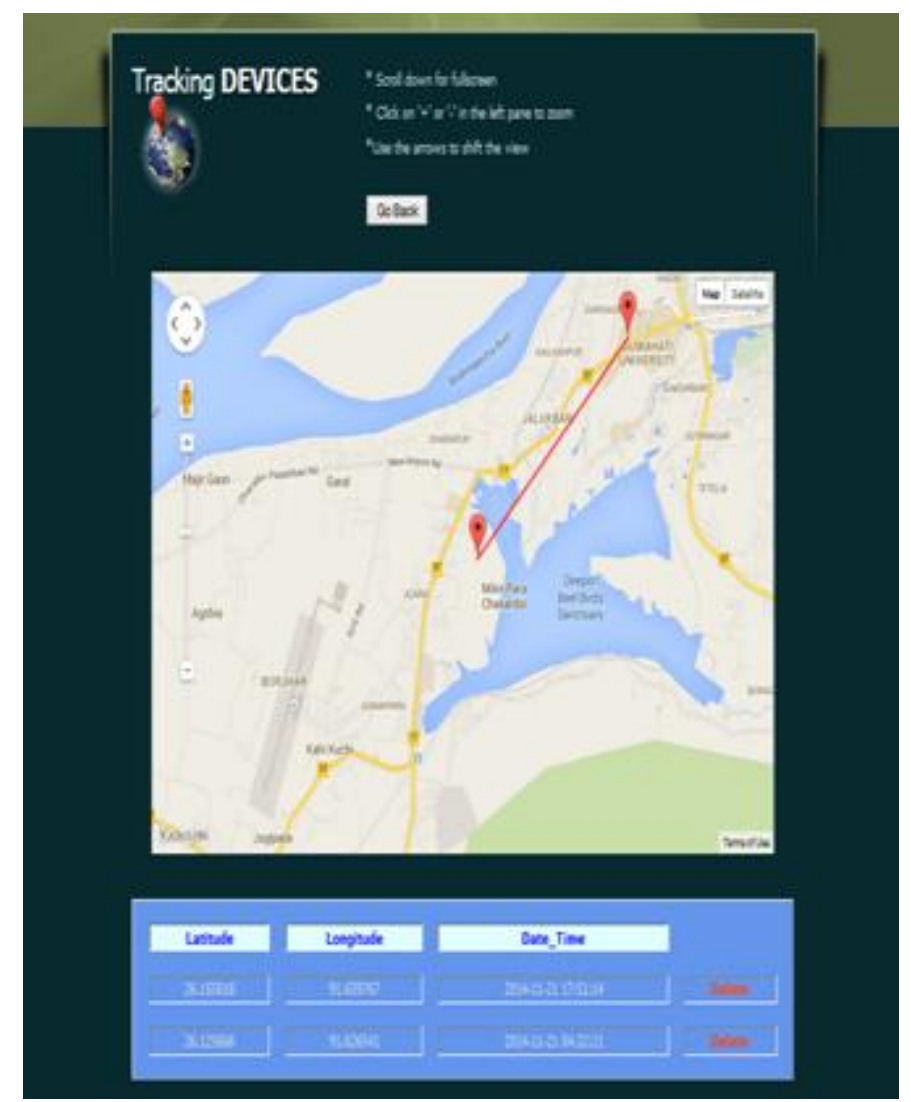

Fig.9(g).Accessing user locations and paths in a Google Map . 


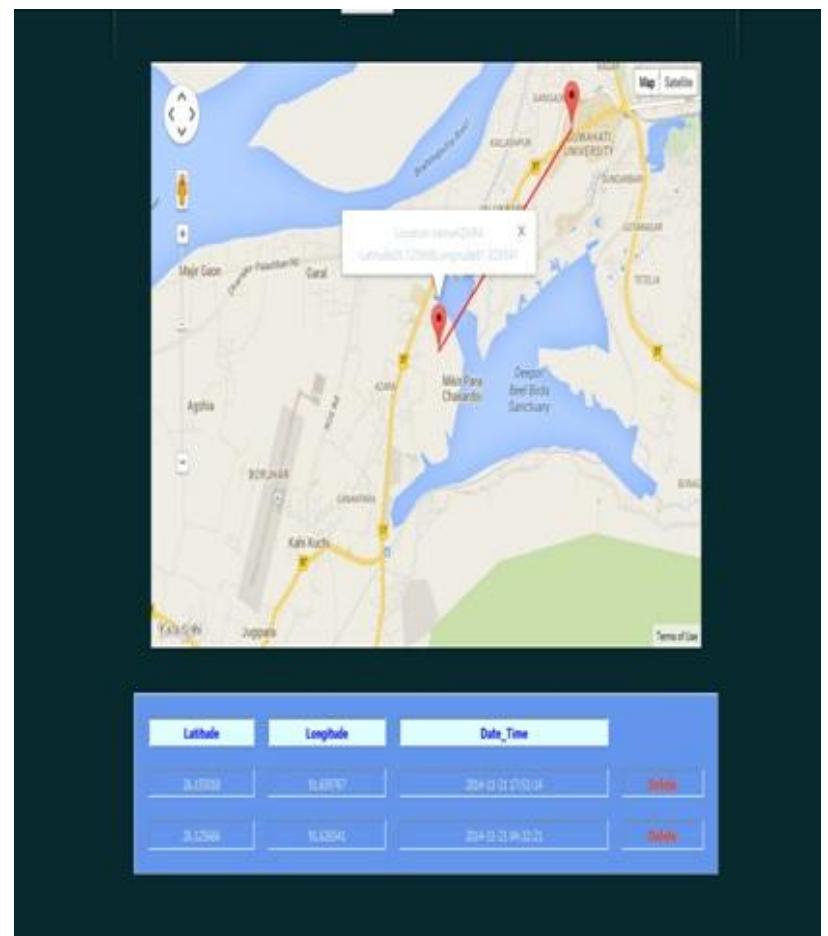

Fig.9(h). Displaying Location details for each user in a Google Map .

\section{REFERENCES}

[1]. K.Virrantaus,J. Markkula, A.Garmash, V.Terziyan, J.Veijalainen, A.Katanosov, and H.Tirri, Developing gissupported location-based services. In Web Information Systems Engineering (2001), IEEE,pp. 66_75.

[2]. http://developer.android.com/tools/devices/index.html

[3]. http://www8.garmin.com/aboutGPS/

[4]. http://www.ibm.com/developerworks/ibm/library/i-lbs 